\title{
Reflexiones sobre el aprovechamiento docente de las nuevas tecnologías docentes por parte de los estudiantes de geografía de la Universidad de Valencia
}

\author{
Pitarch Garrido, María Dolores \\ Albertos Puebla, Juan Miguel
}

Instituto Interuniversitario de Desarrollo Local

Departamento de Geografía. Universidad de Valencia

\begin{abstract}
Resumen:La puesta en marcha de los nuevos Grados de Geografía en las universidades españolas supone la necesidad de incorporar nuevas metodologías didácticas entre las que destaca el uso de las tecnologías de la información y la comunicación (TIC). Al mismo tiempo, los estudiantes universitarios son usuarios habituales, y en muchos casos, experimentados, de estas tecnologías. Las universidades han invertido capitales y recursos humanos importantes para ofrecer tanto a profesores como a estudiantes un acceso fácil y amplio a estas tecnologías. Es el caso de la Universidad de Valencia, en la que desde hace años se dispone de un Aula Virtual, una web institucional, múltiples recursos en red, etc., que facilitan enormemente la comunicación en el entorno universitario. Por otra parte, existen importantísimos recursos de tipo geográfico o territorial (sitios web especializados, bases de datos, visores de cartografía, revistas electrónicas, etc.) disponibles para el público en general. Sin embargo, no conocemos bien cuál es el uso que realizan los estudiantes de Geografía de esas herramientas (en particular, internet) y de los dispositivos que ofrecen las nuevas formas de comunicación para mejorar su proceso de aprendizaje (completar la información de clase, realizar ensayos y trabajos prácticos, etc.). A partir de una encuesta realizada a todos los estudiantes, tanto de Grado como de Licenciatura, se pretende exponer la realidad actual en este tema para el caso de Valencia y reflexionar sobre acciones y métodos didácticos por parte del profesorado que puedan contribuir a una mejor interacción entre las competencias tecnológicas y los métodos de enseñanza apoyados en el uso de las TICs.

Palabras clave: Docencia en Geografía, Innovación docente, Tecnologías de la Información y Comunicación (TICs), Universidad de Valencia.
\end{abstract}

\begin{abstract}
The development of the new Degrees of Geography in the Spanish universities means the need to incorporate new didactic methodologies among which stands out the use of the technologies of information and communication (TIC). At the same time, the university students are habitual users, and in many cases, experienced, of these technologies. The universities have invested capital and human important resources to offer both to teachers and to students an easy and wide access to these technologies. It is the case of the University of Valencia, with a Virtual Classroom, an institutional web, multiple resources in network, etc., that facilitate enormously the communication in the university environment. On the other hand, there exist the most important resources of geographical or territorial type (specialized web sites, databases, visors of cartography, electronic magazines, etc.) available for the public in general. Nevertheless, we do not know well which is the use that the students of Geography make of these tools (especially, Internet) and of the devices that they offer the new forms of communication to improve his learning process (to complete the information of class, to realize tests and practical works, etc.). From a survey realized to all the students, both of Degree and of Master, we try to expose the current reality in this topic for the case of the University of Valencia and to think about actions and didactic methods to contribute to a better interaction between the technological competitions and methods of education rested on the use of the TIC.
\end{abstract}

Key Words: Teaching in Geography, educational Innovation, Technologies of the Information and Communication (TIC), University of Valencia.

(Fecha de recepción: marzo, 2013, y de aceptación: septiembre, 2013)

DOI: 10.7203/DCES.27.2341 


\section{Introducción: El papel de las TIC en la innovación educativa universitaria}

El proceso de convergencia europeo en el que la Universidad española está inserta ha supuesto algunas modificaciones fundamentales en la forma de entender la enseñanza superior. La educación formal tradicional ha quedado obsoleta ante la realidad de una sociedad que cambia a un ritmo desconocido hasta la actualidad. Los contenidos de aprendizaje que han sido definidos por expertos para que los estudiantes adquieran los conocimientos considerados necesarios para superar un nivel educativo superior, han de adaptarse de manera continua a los intereses y necesidades del alumnado, su realidad social y sus perspectivas de futuro, sin perder su solidez científica basada en la propia tradición y evolución de las diferentes disciplinas.

Los países europeos se comprometieron a realizar una serie de cambios en las titulaciones universitarias tendentes a conseguir una mayor homogeneidad, pero a la vez una mayor flexibilidad en los itinerarios de estudios. Los perfiles nuevos para las titulaciones universitarias se basan en las competencias a desarrollar y no solamente en los conceptos a adquirir (Bricall, 2004).

Según Sánchez y Zubillaga (2005), dos han sido los elementos fundamentales que han transformado la educación superior en los últimos años: la integración de las tecnologías de la información y la comunicación en la actividad docente e investigadora y la creación del Espacio Europeo de Educación
Superior (EEES) con las consiguientes acciones de coordinación ante el proceso de convergencia europeo. Ambos elementos se encuentran íntimamente relacionados.

El EEES pone al estudiante en el centro del proceso de enseñanza, lo cual viene unido a un cambio metodológico orientado a potenciar el papel activo del estudiante, la iniciativa y el pensamiento crítico (Esteve, 2009; Trujillo y Raso, 2010). El modelo competencial que otorga el protagonismo al estudiante se ha convertido en la base de los procesos de enseñanza-aprendizaje. El hecho de contabilizar el trabajo del estudiante más allá de la asistencia a clases supone desarrollar un sistema en el que dicha clase es sólo un apoyo más al aprendizaje, pero no el primero ni el único. En este contexto las tecnologías de la comunicación juegan un papel principal, ya que facilitan el desarrollo de las nuevas competencias, en particular del aprendizaje autónomo, tanto por parte de los estudiantes como del profesorado.

La alfabetización digital continúa su ascenso como una habilidad clave para cualquier disciplina y profesión; según el Informe Horizon (The New Media Consortium, 2011), esta meta aún no ha sido alcanzada, aunque hace ya algunos años que se señaló su importancia crítica. Aunque los programas de formación docente universitaria están incluyendo cada vez más cuestiones relacionadas con este tema la realidad es que las tecnologías digitales cambian con una rapidez superior a la que el currículo es capaz de actualizarse. A ello se une el doble reto de proporcionar servicios 
de calidad por parte de las instituciones universitarias y reducir los costes en la medida de lo posible, lo cual está suponiendo la adopción de innovaciones en el campo de la enseñanza superior relacionadas con las tecnologías de la información y la comunicación (TIC) tales como los cursos a distancia o en red. Dada la abrumadora cantidad de novedades tecnológicas y la ingente cantidad de información disponible, es preciso filtrar, organizar e interpretar toda esta avalancha. Este tipo de herramientas y habilidades serán cada vez más estratégicas entre los profesionales dentro y fuera de las universidades (The New Media Consortium, 2011).

El proceso de Bolonia está llevando a las universidades europeas, en particular a las de los países del sur, a transformar la manera de impartir la docencia. El nuevo docente no debe centrarse únicamente en la transmisión de conocimientos, sino que es el alumno quien debe adquirir la capacidad de aprender más que de ser enseñado. El profesor es clave en esta transformación, induciendo a la capacitación del individuo para que sea autónomo ante la búsqueda de información y el consiguiente manejo de las técnicas necesarias para la producción y el alcance del nuevo conocimiento (Trujillo y Raso, 2010; Cos, Meer y Reques, 2011). En este campo, las TIC se configuran como una herramienta clave y con un valor cada vez más importante como parte del aprendizaje basado en las competencias (Joint Quality Initiative, 2004).

Las distintas herramientas desarrolladas por las universidades en los últimos años se basan en internet como espacio articulador de los diferentes ámbitos de aprendizaje (Bosco, 2005). $\mathrm{Su}$ función principal es la gestión y administración de la información y la distribución y comunicación entre profesores y estudiantes. Los campus virtuales son el ejemplo más conocido y difundido.

Uno de los objetivos clave del EEES es la formación a lo largo de toda la vida. En este sentido, es necesario que los individuos aprendan a organizar su propia formación y a intervenir en ella de forma tanto individual como colectiva. En la sociedad de la información y del conocimiento ha de cambiar la actitud frente a la educación.

El Parlamento Europeo y el Consejo (2006) establecen ocho competencias clave para el aprendizaje permanente: comunicación en la lengua materna, comunicación en lenguas extranjeras, competencia en matemáticas, en ciencia y tecnología, competencia digital, aprender a aprender, competencias interpersonales, interculturales y sociales, espíritu de empresa, y expresión cultural. Concretamente, la competencia digital conlleva un uso seguro y crítico de las tecnologías de la sociedad de la información y, por tanto, el dominio de las tecnologías de la información y la comunicación. La clave está en que el estudiante debe ser capaz de seguir con esta formación a lo largo de toda la vida.

El llamado Informe Reflex (ANECA, 2008) señala que el $33 \%$ de los egresados del sistema universitario europeo destacan como punto débil de su carrera la capacidad de utilizar herramientas informáticas. Aunque hay diferen- 
cias según la carrera cursada, en todos los casos, la contribución de los estudios superiores a adquirir o mejorar las competencias en estos temas son muy bajas y la mayor parte de las mismas han sido adquiridas de manera autónoma y no a través de los estudios.

Por su parte, la normativa española, desarrollada en el Real Decreto $1393 / 2007$ por el que se establece la ordenación de las enseñanzas universitarias oficiales, señala que deben proveerse de los medios materiales necesarios, incluidas las nuevas tecnologías, para garantizar el desarrollo de las actividades formativas, observando, según el decreto, los criterios de accesibilidad universal y diseño para todos. Las TIC son, como muchos autores han señalado, un recurso metodológico y no una finalidad educativa, lo cual no le quita importancia, sino más bien la incrementa.

Así pues, entre las propuestas para la renovación de las metodologías docentes en la Universidad, muy amplias y variadas, encuentra su hueco la importancia de las nuevas tecnologías como forma de facilitar recursos didácticos en la red y mejorar el acceso a los mismos por parte de los estudiantes (Ministerio de Educación y Ciencia, 2006). La formación del profesorado en estos temas se considera crucial para conseguir que toda la comunidad universitaria y no sólo una parte de la misma, se comprometa en el proceso de cambio para afrontar el reto de la convergencia europea.

La responsabilidad del profesorado en el uso de las TIC en docencia es clave en cuanto que si no incorpora esta tecno- logía corre en riesgo de quedarse obsoleto en relación a sus alumnos (Vera y Pérez, 2004). Sin embargo, la formación de los profesores en este campo debe tener una fuerte componente didáctica y no sólo tecnológica, con el fin de hacer compatible ambos aspectos: la renovación didáctica y la incorporación de la TIC a la docencia (Bosco, 2005; Imbernon et al, 2011). En definitiva, la clave de la utilidad de las TIC se encuentra en que el profesor actúe como dinamizador y que lo virtual y lo presencial se complementen, no se sustituyan (Cos, Meer y Reques, 2011).

\section{Las TIC en geografía}

A pesar de la importante evolución de las TIC y de su incorporación generalizada en la educación superior a lo largo de las últimas décadas, no parece que las prácticas docentes dominantes en el aula hayan cambiado considerablemente. Según el Libro Blanco, en Geografía, la incorporación de las TIC en la docencia se ha orientado básicamente a las prácticas de las diversas asignaturas que son consideradas aplicadas (Tulla, 2004). Las TIC han ido introduciéndose de manera progresiva, pero acelerada, en la sociedad, generando un nuevo entorno de posibilidades, entre las que se encuentran las relacionadas con la enseñanza superior. Sin embargo, en Geografía, como en otras disciplinas, la introducción de esta innovación en principio no supuso una transformación relevante del modelo pedagógico dominante. La llegada de la tecnología digital y de internet sí ha conseguido generar un cambio que ha 
abierto posibilidades, hasta ahora desconocidas, a la docencia centrada en el alumno, tal como se recomienda en el entorno del EEES (Tulla, 2004).

A pesar de ello, aún existe un desajuste entre la potencialidad de las TIC en el aula de Geografía y la escasa renovación en los procesos pedagógicos o de metodología didáctica. Donde sí han tenido gran impacto ha sido en la comunicación-gestión entre estudiantes y profesores y entre servicios administrativos y estudiantes. Las TIC se han incorporado en la Universidad asociadas a prácticas docentes poco participativas y, en muchos casos, únicamente como forma de transmisión de información del profesorado a los alumnos y no al contrario, sustituyendo el antiguo sistema de fotocopias y diapositivas, por ejemplo. Ello indica que el simple hecho de incorporar tecnología no siempre supone una transformación directa hacia la innovación educativa. Lo que sí es evidente es que las TIC facilitan la flexibilidad en la organización del tiempo y en el uso del espacio, lo cual en los estudios de geografía presenta unas potencialidades enormes.

Cabe añadir que el Libro Blanco de Geografía considera que las nuevas tecnologías multimedia e internet ofrecen al graduado en Geografía posibilidades profesionales importantes, en particular para la divulgación de información y conocimientos. Este campo ofrece en la actualidad rápidas posibilidades de inserción laboral (Tulla, 2004).

El presente artículo analiza el uso de los recursos electrónicos a través de internet de los estudiantes de Geografía, no del profesorado. Este punto de vista, poco estudiado entre los estudiantes de Geografía, se configura como fundamental pues el modelo de enseñanza actual, como preconiza el EEES, está dirigido hacia unos alumnos que ya utilizan las nuevas tecnologías en todas o casi todas sus manifestaciones sociales, aunque no siempre lo hacen para su formación. En un estudio realizado entre los estudiantes de Ciencias Sociales de la Universidad de Alicante se llegó, entre otras, a la constatación de que la relación entre el nivel de conocimientos informáticos y los conocimientos sobre la aplicación de los mismos a la enseñanza es inversa y su grado de conocimiento está muy relacionado con sus necesidades académicas (Vera y Pérez, 2004). El objetivo fundamental del análisis que se presenta a continuación es conocer hasta qué punto los estudiantes, en nuestro caso de Geografía, utilizan los recursos que les ofrece la Universidad a través de internet en la mejora de su relación con la propia universidad y de su proceso de aprendizaje. Se analiza el caso de la Universidad de Valencia ya que nunca con anterioridad se había realizado ningún estudio sobre este tema para este colectivo de estudiantes (titulaciones de Geografía), aparentemente buen conocedor de las ventajas de internet, pero poco dado a su aplicación como parte de su comunicación habitual con el entorno académico. Se trata de una primera incursión, meramente exploratoria, en el análisis de estos aspectos para, en un futuro, ampliar el estudio hacia el uso potencial de las TIC específicas de Geografía en las distintas asignaturas del nuevo grado de Geografía y Medio Ambiente 
que se está empezando a implantar en la Universidad de Valencia y en otras universidades de España.

\section{El uso de las TIC entre los estudiantes de geografía de la Universidad de Valencia}

\section{Conocimientos generales}

Ficha técnica y caracterización de la muestra

Con el fin de conocer las características del comportamiento relacionado con el uso de internet de los estudiantes de Geografía de la Universidad de Valencia, se ha realizado una encuesta.

Dicha encuesta se realizó a todos los estudiantes de Geografía (de todos los cursos) que acudían a clase. La fecha de ejecución de la misma fue el mes de mayo de 2011. La cumplimentación del cuestionario era, como no podía ser de otra manera, voluntaria, aunque la mayoría de los estudiantes que acudían a clase habitualmente se prestaron a rellenarla sin problemas. El tamaño de la muestra puede parecer escaso pero se trata de la mayor parte de los estudiantes que acuden regularmente a clases presenciales. Cabe tomar esta investigación por su carácter heurístico como base para futuros trabajos de este tipo.

Tamaño de la muestra: 77 estudiantes.

Margen de error considerando $\mathrm{p}=0,5$ y un margen de confianza del $95,4 \%$ :

\begin{tabular}{|c|c|c|c|c|}
\hline $\begin{array}{c}\text { Valor de } \mathrm{p} \\
(\% \text { de la } \\
\text { muestra })\end{array}$ & $\begin{array}{c}\text { Margen de } \\
\text { error } \\
(\mathrm{n}=77)\end{array}$ & $\begin{array}{c}\text { Intervalo de } \\
\text { confianza } \\
(\mathrm{n}=77)\end{array}$ & $\begin{array}{c}\text { Margen de } \\
\text { error } \\
(\mathrm{n}=154)\end{array}$ & $\begin{array}{c}\text { Intervalo de } \\
\text { confianza } \\
(\mathrm{n}=154)\end{array}$ \\
\hline 50 & $+/-11.4$ & $38.6-51.4$ & $+/-8.1$ & $41.9-58.1$ \\
\hline
\end{tabular}

La muestra presenta las siguientes características (Cuadro 1A a 1D ):

\section{Cuadro IA}

\begin{tabular}{|c|c|c|c|c|}
\hline \multicolumn{2}{|c|}{ SEXO } & \multicolumn{3}{c|}{ EDAD } \\
\hline Hombres & Mujeres & 18 a 20 años & 20 a 25 años & 25 a 40 años \\
\hline $75 \%$ & $25 \%$ & $63 \%$ & $29 \%$ & $8 \%$ \\
\hline
\end{tabular}

Fuente: Elaboración propia 


\section{Cuadro IB}

\begin{tabular}{|c|c|c|c|c|}
\hline \multirow{2}{*}{ ESTUDIOS EN CURSO } & \multicolumn{3}{|c|}{ LUGAR DE RESIDENCIA } \\
\hline Grado & Licenciatura & Valencia & Área Metropolitana & Otros \\
\hline $73 \%$ & $27 \%$ & $52 \%$ & $16 \%$ & $32 \%$ \\
\hline
\end{tabular}

Fuente: Elaboración propia

\section{Cuadro IC}

\section{TIPO DE RESIDENCIA}

\begin{tabular}{|c|c|c|c|c|}
\hline Familia & Piso de estudiantes & Solo/a & Residencia de estudiantes & En pareja \\
\hline $70 \%$ & $26 \%$ & $1 \%$ & $1 \%$ & $1 \%$ \\
\hline
\end{tabular}

Fuente: Elaboración propia

\section{Cuadro ID}

\begin{tabular}{|c|c|c|c|c|c|}
\hline \multicolumn{2}{|c|}{ TIPO DE PC } & \multicolumn{4}{c|}{ LUGAR DE ACCESO A INTERNET } \\
\hline Sobremesa & Portátil & Ambos & Casa & Facultad & Ambos \\
\hline $21 \%$ & $41 \%$ & $38 \%$ & $54 \%$ & $1 \%$ & $46 \%$ \\
\hline
\end{tabular}

Fuente: Elaboración propia

La mayor parte de los estudiantes de Geografía de la Universidad de Valencia son hombres. Hay muy pocas mujeres, y su cifra se reduce cuanto más jóvenes son las generaciones, es decir, en los estudios de Grado son aún más minoritarias. Las edades de los estudiantes están comprendidas entre los 18 y los 25. Muy pocos son los mayores de esta edad que se decantan por seguir estos estudios. Es significativo que la mayor parte de los que han respondido a la encuesta sean estudiantes de Grado, siendo muchos menos los estudiantes de licenciatura que han deseado contestar (y que han acudido a clase para hacerlo). 
La mayor parte de los estudiantes de Geografía residen en la ciudad de Valencia o en su área metropolitana. Los que residen a mayor distancia, algo más del $30 \%$, se encuentran generalmente a menos de una hora de distancia de la facultad, en la provincia de Valencia. La mayoría viven con sus familias tanto en la ciudad como fuera de ella y sólo un $26 \%$ comparten piso con otros estudiantes. Otras formas de residencia son muy minoritarias.

La mayoría de los estudiantes de Geografía poseen un ordenador portátil, o bien como único ordenador o bien además del de sobremesa. El 79\% de los encuestados declararon esta situación. Tanto la forma de residencia como el tipo de ordenador disponible pueden ser factores que expliquen la facilidad o dificultad de acceder a internet. Sin embargo, la inmensa mayoría de los encuestados acceden a la red desde casa, sólo el $1 \%$ lo hace únicamente en la facultad, en la que el wi-fi funciona desde hace unos años, además de los laboratorios y aulas de informática, todos conectados a internet.

\section{El uso de internet por parte de los estudiantes}

Como hemos señalado, todos los estudiantes encuestados tienen ordenador y todos ellos también acceso a internet, en casa y en la facultad. La mayoría, el $76 \%$ accede diariamente a la red por algún motivo (Cuadro II). Destacan algo por encima los hombres: el $71 \%$ acceden dos o más veces al día, mientras que el $68 \%$ de las mujeres lo hacen con esa frecuencia (Cuadro III).

\section{Cuadro II \\ Frecuencia de acceso a internet \\ (\% horizontal)}

\begin{tabular}{lccc}
\hline & Hombre & Mujer & Total \\
\hline 2 o más veces al día & 76 & 24 & 100 \\
1 vez al día & 74 & 26 & 100 \\
Cada 2 días o más & 67 & 33 & 100 \\
\hline
\end{tabular}

Fuente: Elaboración propia

\section{Cuadro III}

Frecuencia de acceso a internet (\% vertical)

\begin{tabular}{lcc}
\hline & Hombre & Mujer \\
\hline 2 o más veces al día & 71 & 68 \\
1 vez al día & 25 & 27 \\
Cada 2 días o más & 4 & 5 \\
TOTAL & 100 & 100 \\
\hline
\end{tabular}

Fuente: Elaboración propia

Cabe destacar, a considerar con las anteriores características, la frecuencia de acceso según el tipo de estudios (Cuadro IV). Los estudiantes de Grado, más jóvenes, en general, acceden con mayor frecuencia a internet, varias veces al día, mientras que los estudiantes de Licenciatura acceden, con más frecuencia que los anteriores, al menos una vez al día. En definitiva, los estudiantes de Geografía, en general, tienen una relación con internet que es cada vez más intensa, formando parte de su forma de comunicarse en su vida diaria (Gráfica 1). 
Cuadro IV

Frecuencia de acceso a internet según tipo de estudios en curso (grado o licenciatura)

\begin{tabular}{lccc}
\hline & Grado & Licenc. & Total \\
\hline 2 o más veces al día & 77 & 23 & 100 \\
1 vez al día & 58 & 42 & 100 \\
Cada 2 días o más & 100 & 0 & 100 \\
\hline
\end{tabular}

Fuente: Elaboración propia

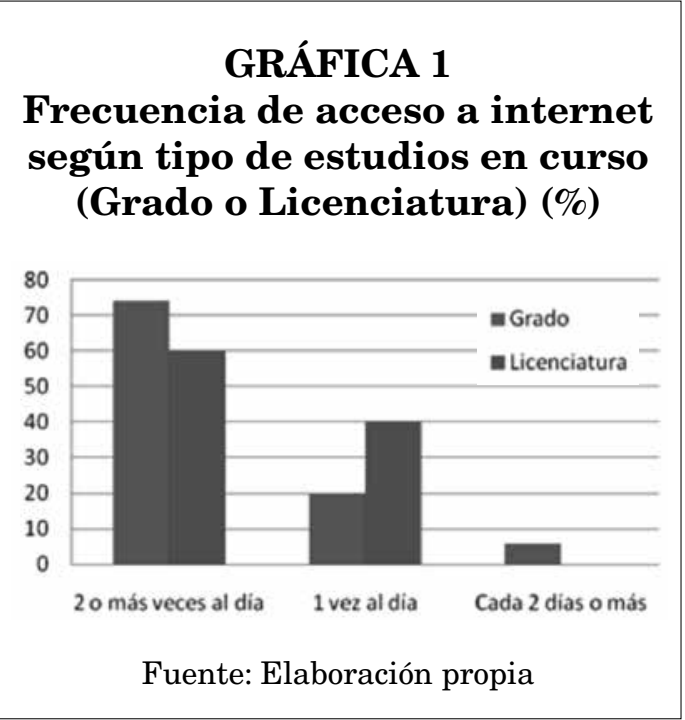

Son los estudiantes que viven con sus familias los que presentan mayores posibilidades de acceso a internet desde casa. Esta situación les da una mayor flexibilidad a la hora de acceder a materiales de estudio o de realizar cualquier tipo de gestión universitaria a través de la red. Los estudiantes que viven en pisos compartidos, también tienen posibilidades de acceso en casa, aunque son poco más de la mitad de los encuestados los que expresan dicha posibilidad (Cuadros V y VI). Mayoritariamente señalan su acceso tanto en casa como en la Universidad, por encima de lo declarado por los estudiantes que viven con sus familias.

\begin{tabular}{|c|c|c|c|c|}
\hline $\begin{array}{r}\text { Lugar de } \\
\text { tipo de }\end{array}$ & $\begin{array}{l}\mathrm{Cu} \\
\text { acces } \\
\text { reside }\end{array}$ & ncia ( & $\begin{array}{l}\text { rnet } \\
\text { vert }\end{array}$ & $\begin{array}{l}\text { según } \\
\text { ical) }\end{array}$ \\
\hline & $\begin{array}{c}\text { Con la } \\
\text { familia }\end{array}$ & $\begin{array}{l}\text { Piso de } \\
\text { estud. }\end{array}$ & Solo/a & $\begin{array}{l}\text { Otra } \\
\text { modal. }\end{array}$ \\
\hline En casa & 62 & 37 & 0 & 33 \\
\hline En la Univer & 0 & 5 & 0 & 0 \\
\hline Ambas & 38 & 58 & 100 & 67 \\
\hline TOTAL & 100 & 100 & 100 & 100 \\
\hline
\end{tabular}

Fuente: Elaboración propia

\section{Cuadro VI}

Lugar de acceso a internet según tipo de residencia (\% horizontal)

\begin{tabular}{lccccc}
\hline \multicolumn{2}{r}{$\begin{array}{r}\text { Con la } \\
\text { familia }\end{array}$} & $\begin{array}{c}\text { Piso de } \\
\text { estud. }\end{array}$ & Solo/a & Otra \\
modal. & Total \\
\hline En casa & 80 & 17 & 0 & 3 & 100 \\
En la Univ & 0 & 100 & 0 & 0 & 100 \\
Ambas & 59 & 32 & 3 & 6 & 100 \\
\hline
\end{tabular}

Fuente: Elaboración propia

En la lógica anterior, también los estudiantes que viven en pisos compartidos poseen ordenador portátil, en mayor porcentaje que los que viven con sus familias. Ello les facilita la movilidad, tanto de residencia (fines de semana o periodos vacacionales en sus lugares de origen) como en el ámbito universitario. El mayor acceso a internet en la universidad por parte de los estudiantes en pisos compartidos se explica también por su disponibilidad mayor de ordenador portátil (Cuadro VII). 


\section{Cuadro VII \\ Tipo de ordenador según tipo de residencia}

\begin{tabular}{lcccc}
\hline & $\begin{array}{c}\text { Con la } \\
\text { familia }\end{array}$ & $\begin{array}{c}\text { Piso de } \\
\text { estud. }\end{array}$ & Solo/a & Otra \\
modal.
\end{tabular}

Fuente: Elaboración propia

Por último, el hecho de disponer de una web propia supone un indicio de interés por las TIC y de conocimientos técnicos más allá de los meramente consultivos. Además, indica también una capacidad de crear contenidos, aunque sean de tipo personal, para publicar de manera abierta a través de internet. Ello será de gran importancia y presenta enormes posibilidades en la formación y autoaprendizaje.

Son, precisamente, los estudiantes de Licenciatura los que disponen de

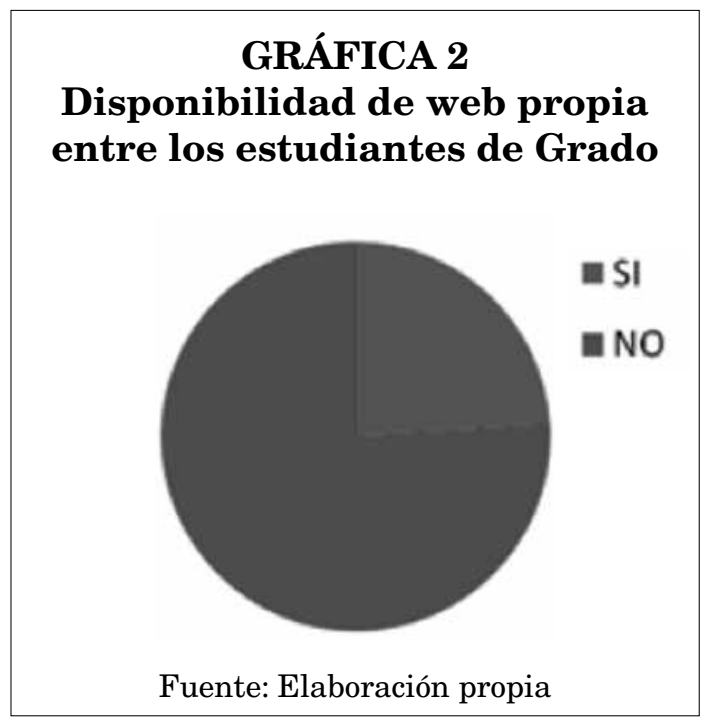

web propia (Gráficas 2 y 3 ). Se trata pues de un indicio de madurez personal y científica. Las diferencias no vienen asociadas a las posibilidades de acceso a internet sino a dos elementos: en primer lugar, la aparición y generalización de las redes sociales, utilizadas por los más jóvenes como forma de relacionarse y mostrar su perfil al mundo, y, en segundo lugar, la capacidad de crear contenidos, lo cual parece asociado a la edad y a la adquisición de conocimientos técnicos y científicos a lo largo de la carrera universitaria. Sin embargo, las posibilidades de creación de contenidos unidas a las innovaciones tecnológicas que están apareciendo en el mercado constantemente y de manera cada vez más económica, puede hacernos prever una importante mejora de la capacidad de los alumnos más jóvenes, los de Grado, ya completamente integrados en el mundo virtual, que en poco tiempo superarán a las generaciones anteriores en el manejo de este tipo de aplicaciones, en particular la web 2.0 y, en un

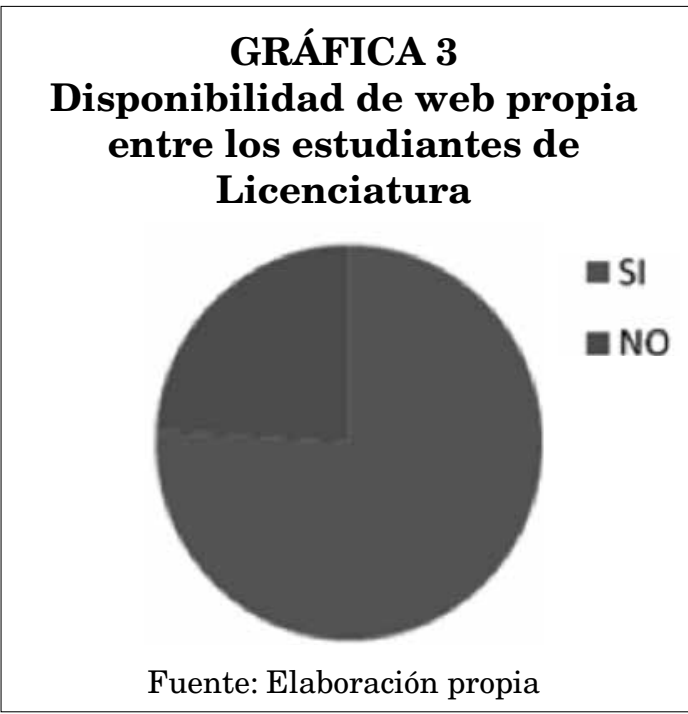


futuro no muy lejano la web 3.0. Se abre un mundo de posibilidades didácticas que no deben desaprovecharse.

Los recursos electrónicos en la vida universitaria

La Universidad de Valencia, como todas, ha incorporado las TIC en la vida académica, incluyendo los aspectos relacionados con la investigación y con la gestión, y ofrece una serie de servicios vía internet orientados especialmente importantes a los estudiantes. Entre ellos destacan algunos que han constituido la base de la encuesta: el Aula Virtual, la web de la Biblioteca, la web del Servicio de Estudiantes y la web de los departamentos universitarios.

Todos los estudiantes encuestados conocen el servicio de Aula Virtual de la Universitat de València y todos ellos lo utilizan. El Aula Virtual responde al modelo de Campus Virtual desarrollado en diferentes entornos pero con la misma finalidad en las universidades tanto españolas como europeas.

El Aula Virtual de la Universidad de Valencia se organiza por asignaturas y suele prepararse expresamente para apoyar el aprendizaje del grupo matriculado. Forma parte de una propuesta de enseñanza más amplia y no sustituye la asistencia a las clases.

Los estudiantes acceden al aula virtual cada día, tanto hombres como mujeres y tanto alumnos de Grado como de Licenciatura (Cuadro VIII). Estos elevados porcentajes reflejan la normalidad con la que el Aula Virtual se ha incorporado en el proceso de aprendizaje. Aún algunos estudiantes sólo acceden cuando es imprescindible y reciben algún tipo de aviso, pero son minoría (Cuadro IX).

\section{Cuadro VIII \\ Uso del aula virtual según sexo ¿Cuándo?}

\begin{tabular}{lcc}
\hline & Hombre & Mujer \\
\hline Cuando llega un aviso al email & 14 & 0 \\
Cuando lo indica el profesor & 2 & 10 \\
Cada día & 84 & 90 \\
TOTAL & 100 & 100 \\
\hline
\end{tabular}

Fuente: Elaboración propia

\begin{tabular}{|c|c|c|}
\hline $\begin{array}{r}\text { Cuadro IX } \\
\text { Uso del aula virtual s } \\
\text { estudios en c } \\
\text { ¿Cuándo? }\end{array}$ & $\begin{array}{l}\text { gún ti } \\
\text { Irso }\end{array}$ & o de \\
\hline & Grado & Licenc. \\
\hline Cuando llega un aviso al email & 7 & 15 \\
\hline Cuando lo indica el profesor & 6 & 0 \\
\hline Cada día & 87 & 85 \\
\hline TOTAL & 100 & 100 \\
\hline
\end{tabular}

Fuente: Elaboración propia

La experiencia con el Aula Virtual en otras universidades (Badia et al., 2006) coincide, en gran parte, con la de la Universidad de Valencia. En ocasiones, los estudiantes consideran que con utilizar el entorno del Aula Virtual para conseguir los materiales que presenta el profesor en clase y enviar los resultados de trabajos o prácticas, es suficiente para alcanzar el mínimo necesario para superar la asignatura. Esta convicción 
se traduce en una asistencia relativamente reducida en el aula y unos resultados finales decepcionantes. Se dan claras diferencias entre los estudiantes que asisten regularmente a las clases en el aula y aquellos que sólo lo hacen de manera esporádica. El Aula Virtual no suple, en ningún caso, la presencialidad.

El uso que los estudiantes de Geografía hacen del Aula Virtual está muy relacionado con las prácticas docentes tradicionales (Cuadro X y Gráfica 4). El 97\% declara usar esta plataforma para descargarse los documentos o recursos que el profesor previamente ha facilitado mediante su búsqueda, preparación y colocación en el Aula Virtual. El 84\% señala que utiliza la plataforma para la evaluación de alguna asignatura y el $78 \%$ para la realización de prácticas. Estos tres usos principales están asociados al trabajo del profesor y no del estudiante. Es el primero quien propone a través del Aula Virtual una serie de documentos y/o ejercicios al igual que lo hace en la clase presencial. El trabajo ahora es doble, pero facilita el acceso de estudiantes que no pueden asistir a clase o de aquellos que, asistiendo, evitan tomar notas o buscar la documentación propuesta por el profesor, ya que todo ello se encuentra a su disposición en el Aula Virtual. Se trata, para los estudiantes de Geografía de una plataforma "facilitadora", pero no de una web de relaciones o de intercambio de conocimientos, opiniones o valoraciones de la asignatura como se aprecia en que sólo el $4 \%$ la utilizan para el contacto con compañeros y el $18 \%$ para la realización de exámenes o tests (esto último también muy ligado a la voluntad del profesor).

\section{Cuadro X Uso del aula virtual (\% de respuestas positivas)}

¿Para qué?

Recursos o documentos que indica

el profesor 97

Ejercicios 0 prácticas

Exámenes 0 tests

18

Contacto con profesores

48

Contacto con compañeros

4

Otros usos

Uso del Aula Virtual en la evaluación

de alguna asignatura

84

Fuente: Elaboración propia

Cabe preguntarse si los materiales ofrecidos por el profesor como Recursos o en las webs de las asignaturas no desempeñan el mismo papel que los libros en la biblioteca tradicional. La diferencia fundamental se encuentra en el acceso y en su carácter multimedia, lo que les da ciertas ventajas como que es más barato (no hay que gastarse el dinero en comprar libros ni en fotocopias) y está disponible para todos.

En este sentido, funciona también la web de la Biblioteca universitaria para los estudiantes de Geografía. Alrededor del $25 \%$ de los estudiantes no conocen los servicios de la biblioteca de la Universidad a través de internet. El 75\% sí los conoce y los utiliza (Cuadro XI).

La web de la Biblioteca de la Universidad presenta una colección de materiales digitales alojados y controlados, independientemente de su procedencia. Además, ofrece una serie de servicios que los estudiantes pueden realizar sin tener que desplazarse hasta las biblio- 


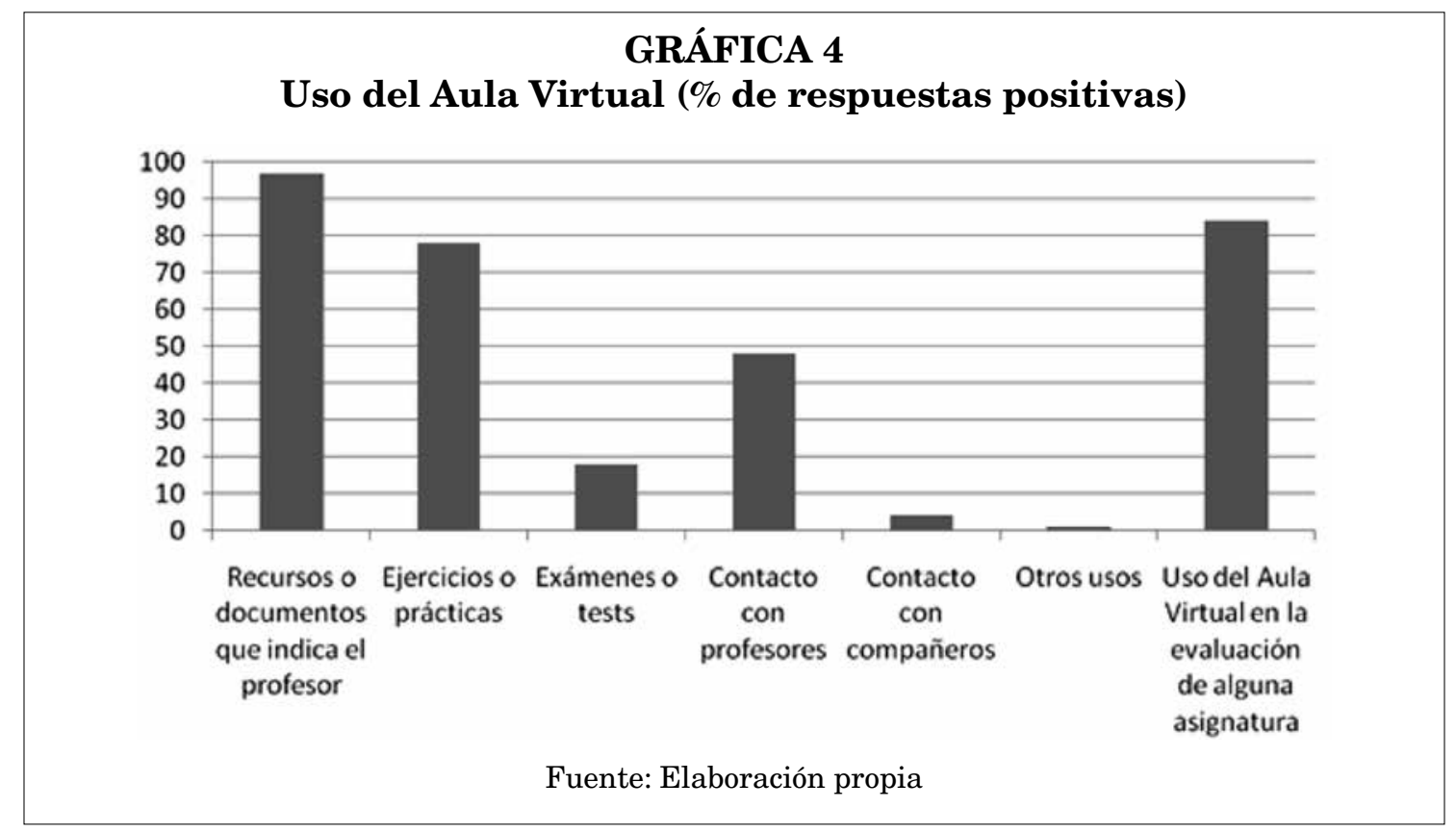

tecas. A pesar de ello, la mayoría utilizan esta web para aspectos prácticos, es decir para realizar algún tipo de trabajo y/o por indicación del profesor, lo cual significa que acuden a ella esporádicamente y no de manera regular como parte de su proceso de aprendizaje para completar información de clase.

\section{Cuadro XI}

\section{Uso de la web de la biblioteca según sexo ¿Cuándo?}

\begin{tabular}{lcc}
\hline & Hombre & Mujer \\
\hline Diariamente & 0 & 0 \\
Una vez a la semana & 20 & 17 \\
Una vez al mes & 11 & 0 \\
Cuando lo indica el profesor & 13 & 6 \\
Cuando he de realizar un trabajo & 32 & 50 \\
Nunca & 24 & 28 \\
TOTAL & 100 & 100 \\
\hline
\end{tabular}

Fuente: Elaboración propia
Más de la mitad de los estudiantes encuestados señalan que utilizan la web de la biblioteca para acceder a libros-e y acuden a la biblioteca por su oferta de wi-fi. El acceso a revistas electrónicas es minoritario, y más aún al fondo digital antiguo (Gráfica 5).

Por otra parte, el $40 \%$ de los estudiantes encuestados declaran conocer el Servei d'Estudiants. Dado que es la parte del portal web de la universidad especialmente diseñado para informar a los estudiantes, no es una cifra demasiado elevada, o al menos es inferior a lo deseable. Esta web proporciona a los estudiantes información fundamental en temas tan diversos como las becas o las posibilidades de realizar actividades deportivas en los campus universitarios.

La mayor parte de los estudiantes de Geografía que conocen la web la uti- 


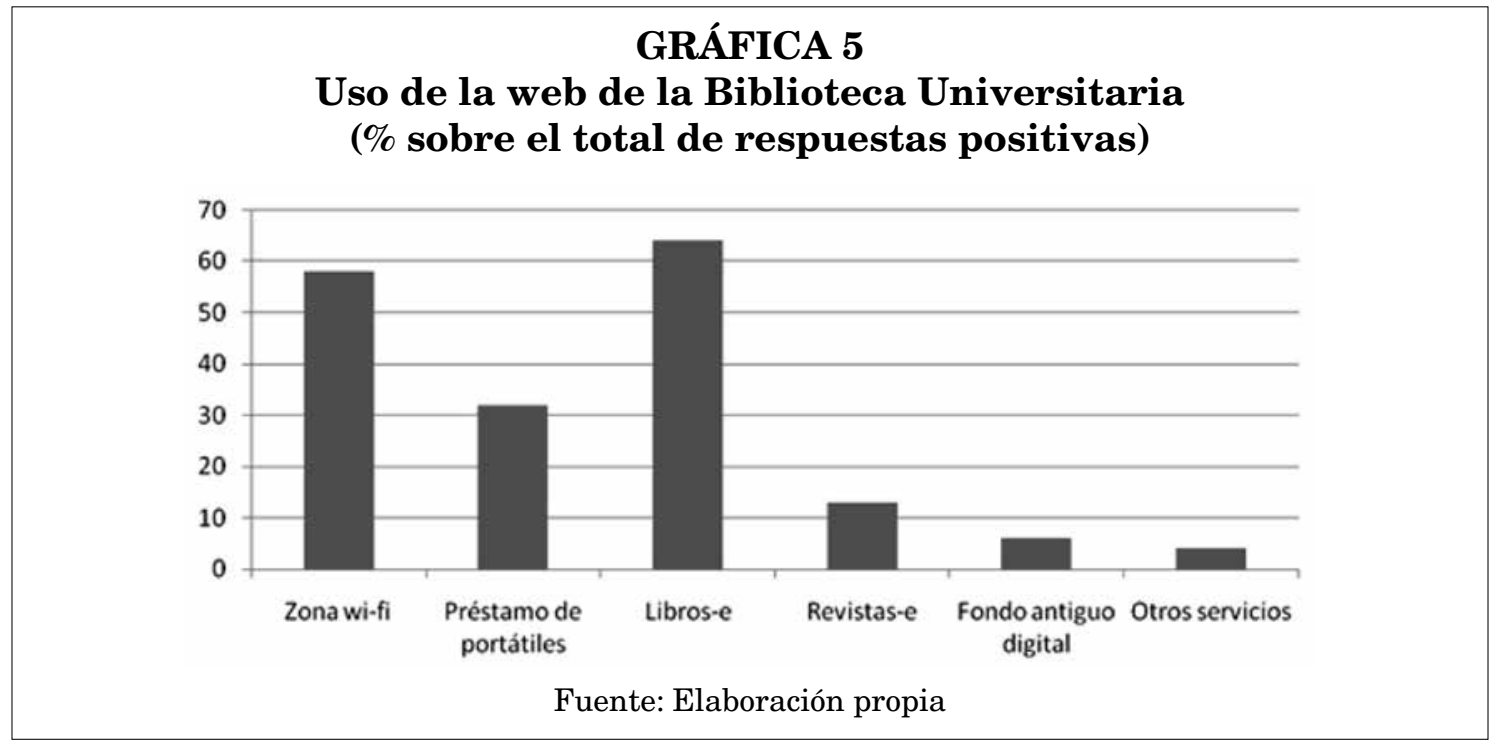

lizan precisamente, como se aprecia en la gráfica 6, para obtener información sobre becas y ayudas (el $81 \%$ ), en menor medida la utilizan también para obtener información académica (68\%) y sobre los grados y postgrados que se están poniendo en marcha en la univer- sidad (52\%). Es mucho menor, apenas el $6 \%$ de los encuestados que conocen la web, el número de los que acceden buscando formación complementaria. De nuevo, el tema de la autoformación queda relegado a un puesto poco significativo.

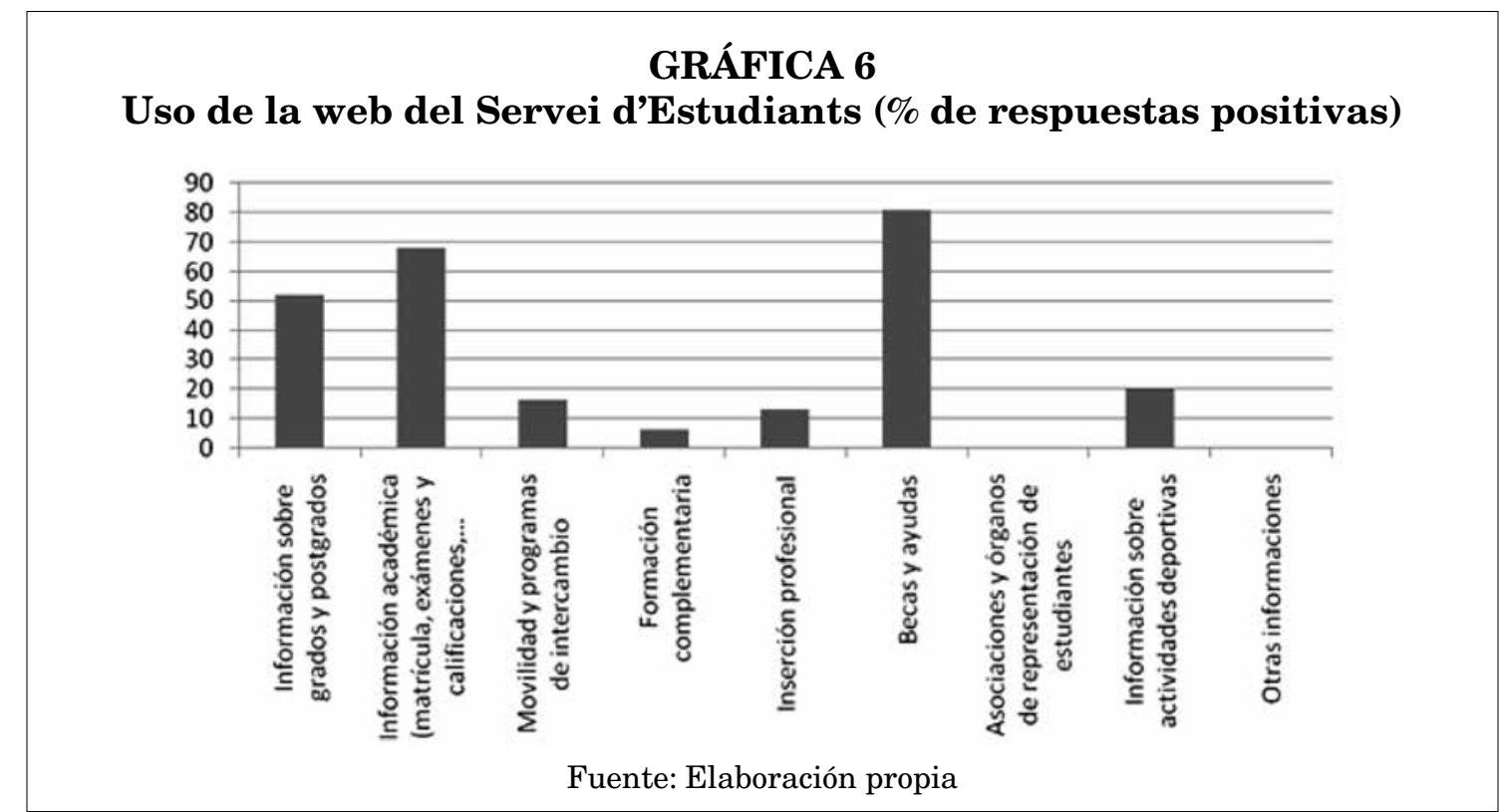


Por último, es de destacar que el 70\% de los encuestados conocen la web del Departamento de Geografía. Inserta en la estructura general de la Universidad, esta web, como las demás webs departamentales, es mantenida por el propio departamento, lo cual ofrece interesantes posibilidades de acercarse a los estudiantes que se han decantado por los estudios de Geografía en particular.

La mayor parte de los estudiantes encuestados que declaran conocer esta web, el $70 \%$, la utilizan fundamentalmente para acceder a las guías docentes de las asignaturas (Gráfica 7). El departamento mantiene actualizados estos ficheros que se pueden descargar en formato pdf, aunque a ellos también se puede acceder a través del Servicio de Estudiantes y de otros apartados de la web general de la Universidad. Sin embargo, la facilidad de uso de esta web y el hecho de que se dirija específica- mente a los estudiantes de geografía, explican su popularidad.

La información sobre los profesores, tanto sus horarios de tutorías como de clases, su correo electrónico y algunos datos más sobre líneas de investigación, es también demandada por los estudiantes. El $45 \%$ acceden a esta información. Las salidas profesionales y los planes de estudio también son consultados en la web del Departamento de Geografía.

Llama la atención el escaso interés por la investigación, el laboratorio de SIG e incluso la revista del Departamento, completamente digitalizada y accesible a través de esta web. De nuevo, las aplicaciones que pueden ayudar a dar un paso más hacia el adecuado uso de las TIC en docencia, tal como señalan los estudios sobre el tema y las propuestas del EEES, son las menos usadas. La capacidad de crear información y que ésta se transforme

\section{GRÁFICA 7 \\ Uso de la web del Departamento de Geografía (\% de respuestas positivas)}

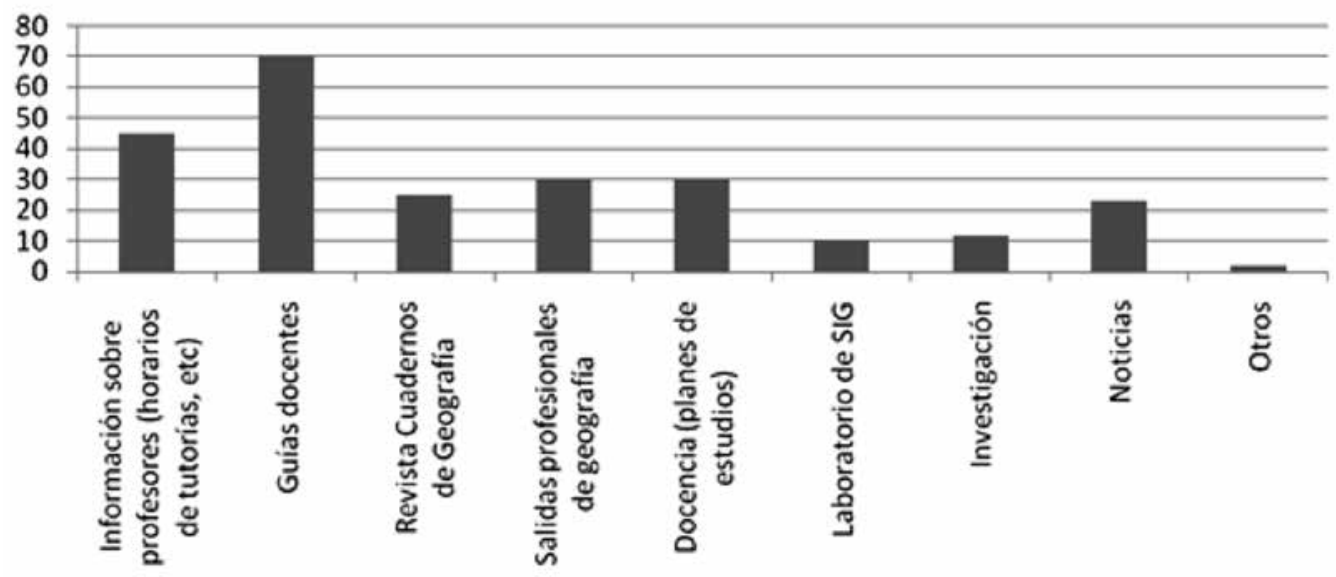

Fuente: Elaboración propia 
en conocimiento, aún es limitada entre los estudiantes de Geografía.

WWW y la relación alumnos-profesores

El correo electrónico ha supuesto un cambio importante en la relación entre el profesor y el alumno. El primero se encuentra siempre disponible, dentro y fuera de su horario laboral y lectivo, e incluso fuera de su horario de tutorías. Los límites los pone el propio profesor, pero a veces no es sencillo. Los estudiantes de Geografía encuestados valoran muy positivamente la posibilidad de contactar con los profesores a través del correo electrónico (Gráfica 8). El 87\% de los encuestados contactan alguna vez por correo electrónico con los profesores y la razón fundamental es la comodidad personal, es decir, la posibilidad de contactar en cualquier momento y lugar con el profesor.

Muy pocos lo hacen porque no puedan acudir personalmente a las tutorías, apenas el $13 \%$ lo señalan como argumento principal para contactar vía correo electrónico. Tampoco es una preferencia del profesor ni éste se ausenta del despacho en horario de tutorías.

Destaca que muchos estudiantes, el $25 \%$ lo señalan en segundo lugar y el $32 \%$ en tercer lugar, indican que el profesor siempre contesta a sus cuestiones a través del correo electrónico, lo cual indica una buena disposición de los profesores del departamento hacia esta forma de comunicación con el alumno.

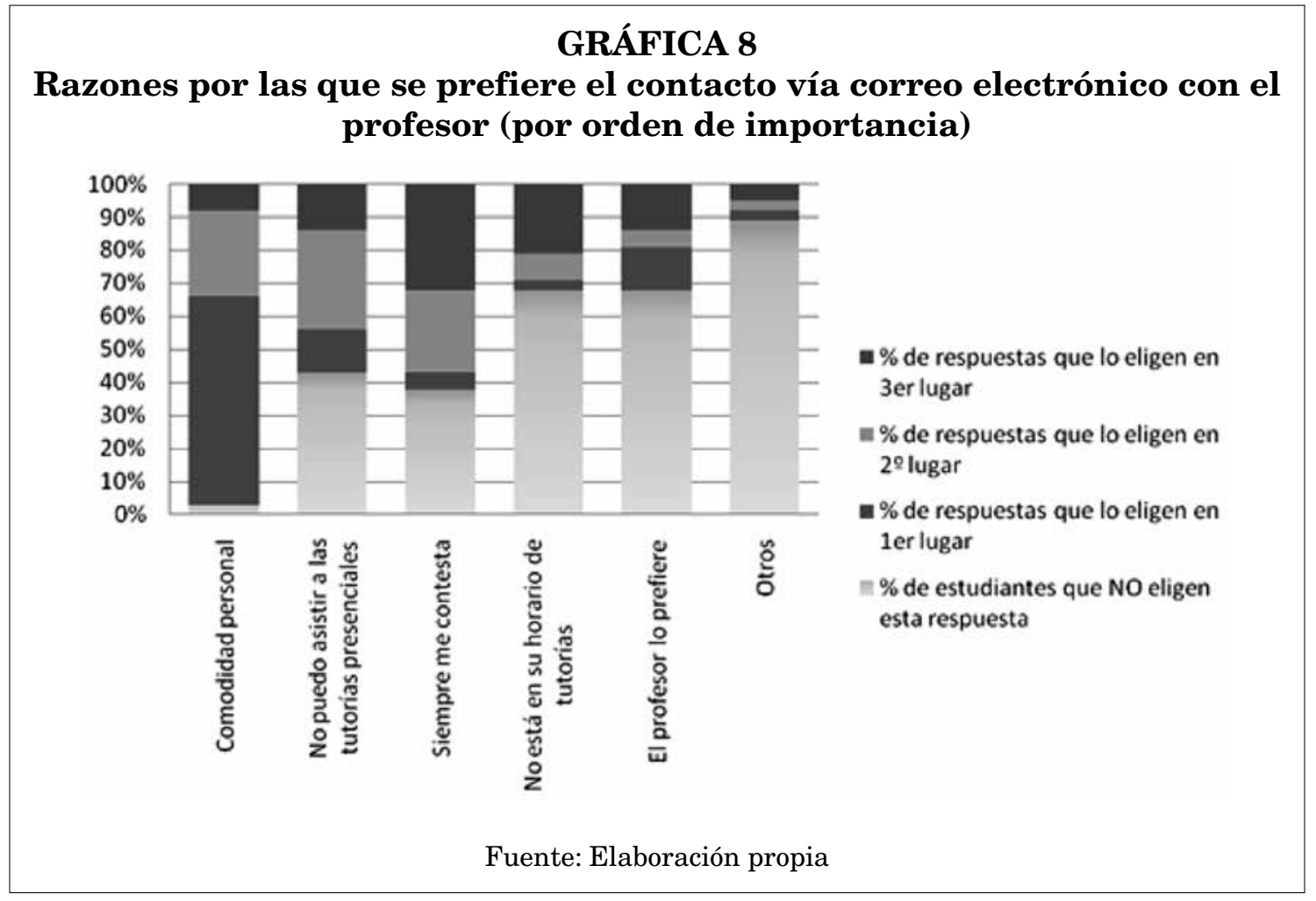


Entre los "otros" motivos que explican la preferencia por usar el correo electrónico para comunicarse con el profesor, se encuentran la rapidez y la posibilidad de dirigirse a ellos fuera del horario de tutorías. Ambas razones clave para entender el uso que los estudiantes esperan de las herramientas web.

Las nuevas formas que ofrecen las TIC permiten el intercambio y el trabajo colaborativo de profesores y alumnos. Las tutorías virtuales son un ejemplo de ello aún muy poco utilizadas en Geografía.

\section{Valoración y propuestas}

Los estudiantes de Geografía encuestados confirman en su valoración de los diversos temas relacionados con el uso de internet para la docencia las cuestiones que han ido saliendo con anterioridad. Para ellos los elementos más importantes son el Aula Virtual, con un $88 \%$ de las respuestas, el correo electrónico del profesor, con un $80 \%$ y el acceso a internet en la facultad (wi-fi), con un 76\% (Gráfica 9). Tienen poca importancia los foros de discusión y, en menor medida, las tutorías virtuales, la web del departamento de Geografía y las webs de las asignaturas. Alrededor de tres cuartas partes de los estudiantes encuestados consideran estas cuestiones poco o nada importantes.

La relevancia que dan al entorno del Aula Virtual, así como al correo electrónico de los profesores se debe a temas prácticos y de utilidad inmediata para superar las asignaturas. El contacto con los profesores de manera cómoda es importante para ellos y, sobre todo, el conocer los materiales que formarán parte de la evaluación aún lo es más. Un foro de discusión, por el contrario, sería una plataforma adecuada para el intercambio de conocimientos sobre la asignatura y para la propuesta de temas de debate y estudio. Sin embargo, no sólo es una herramienta muy poco utilizada, sino que también, en parte como consecuencia de ello, es poco valorada.

Según lo señalado hasta ahora, parece seguir imperando en la docencia de la Geografía la clase tradicional, basada en la impartición de información y conocimientos por parte del profesor, pero esta vez utilizando las TIC, mientras que el alumno asume un papel pasivo y cómodo en el proceso de enseñanza.

A pesar de que la realidad práctica parece imponerse, hay todavía mejoras posibles que debemos implementar para optimizar el uso de las TIC en la docencia. Entre los elementos TIC que podrían incorporarse a la docencia, los estudiantes encuestados señalan la importancia de los apuntes de la asignatura (lo que confirma lo señalado anteriormente), considerados bastante importantes por el $96 \%$, pero destacan también la importancia de materiales gráficos disponibles en internet, acceso a bases cartográficas y ejercicios de autoevaluación (Gráfica 10). Entre los otros aspectos a considerar, los estudiantes no contestan cuestiones concretas sino "cualquier información útil". Ello nos confirma, de nuevo, el uso casi exclusivo de las herramientas disponibles para la obtención información pero no para la generación de la misma.

En este sentido, aún hay un extenso campo para trabajar con las TIC en el 


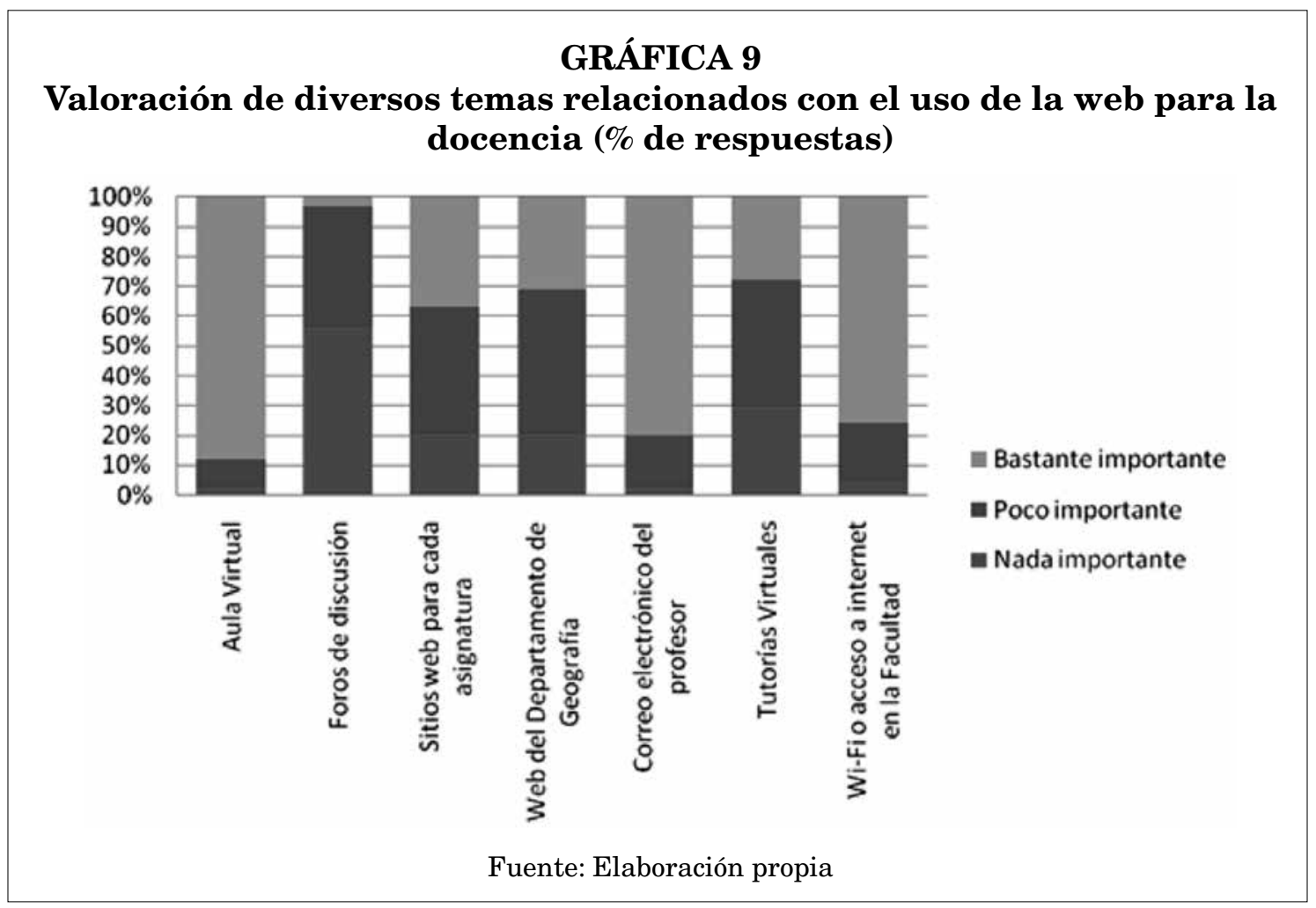

ámbito de la docencia de la Geografía. La cuestión es ir hacia métodos que permitan ayudar al profesor a capacitar al alumno para el trabajo en equipo, la creación de conocimiento y la posibilidad de compartir con los demás ese conocimiento.

\section{Conclusiones: potencialidades de} las TIC en la docencia universitaria de geografía

Está claro que existe una relación muy estrecha entre el modelo educativo vinculado al EEES y la integración de las TIC en la docencia universitaria. El uso de las TIC forma parte de esta renovación, pues se trata de herramientas generalizadas entre los estudiantes que están cambiando sus entornos personales y de aprendizaje, generando una nueva forma de acometer el aprendizaje de competencias. A pesar de ello, la relación no es explícita, pues no se menciona como elemento estratégico en ningún documento sobre los nuevos grados y postgrados de Geografía, razón por la cual nos pareció interesante emprender un estudio exploratorio como el aquí presentado. Lo que sí se observa es una tendencia general a desarrollar acciones en las que el acceso a la información a través de internet tiene un peso importante en su diseño o en su aplicación. En Geografía se ha entendido que el uso de las TIC en docencia forma parte de las medidas orientadas a faci- 


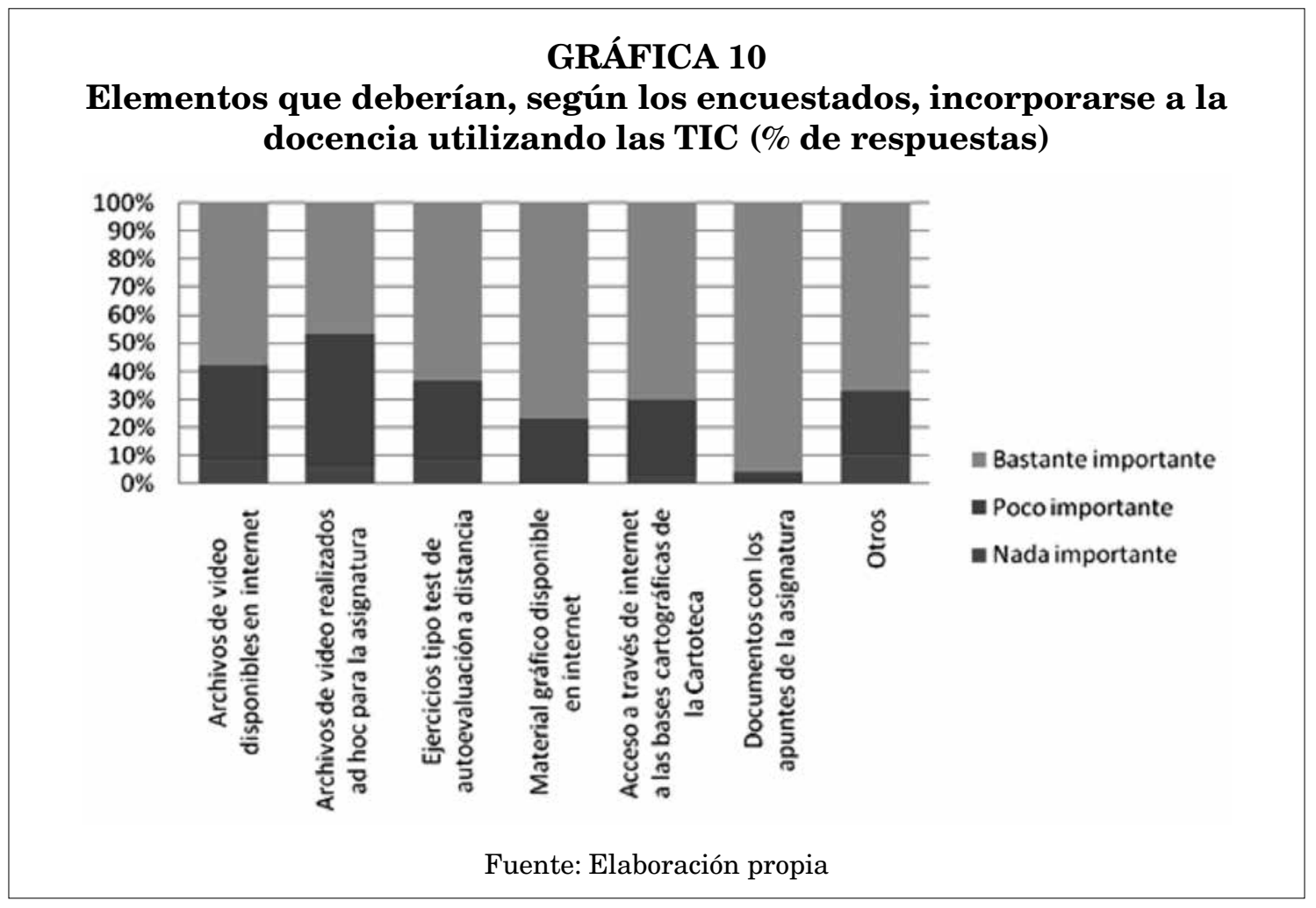

litar el proceso de integración de nuestra disciplina académica en el EEES. El desarrollo del Aula Virtual (por parte de la Universidad), la web del departamento de Geografía y webs específicas para algunas asignaturas forman parte de este proceso, que, si bien no es exclusivo para Geografía, su uso en docencia es un primer aspecto a considerar para conocer la potencia real que tienen para los futuros geógrafos y que, en principio, como se señaló al comienzo de este artículo, se intuía débil.

La clave está en encontrar metodologías didácticas en Geografía que optimicen el uso de las TIC, en particular de los recursos ofrecidos a través de internet. En este sentido, es preciso orien- tar los esfuerzos hacia el diseño de una formación para el uso de dichos recursos en el contexto de una comunidad de aprendizaje que desarrolle procesos colaborativos capaces de crear materiales y formas de enseñanza de calidad.

La gran ventaja que ofrece el uso de internet en docencia es la facilidad de acceso a los recursos, clave para potenciar el trabajo autónomo y la autoevaluación. A pesar de ello, las TIC pueden facilitar el cambio en la forma de entender la enseñanza, pero no pueden producirlo.

La gran ventaja que ofrece el uso de internet en docencia es la facilidad de acceso a los recursos, clave para potenciar el trabajo autónomo y la autoeva- 
luación. A pesar de ello, las TIC pueden facilitar el cambio en la forma de entender la enseñanza, pero no pueden producirlo. Esto parece estar ocurriendo, según nuestro análisis, en la docencia universitaria de Geografía, lo que implica que sea fundamental el apoyo a las iniciativas de mejora docente que incorporen recursos en internet y nuevas tecnologías en general, pues hasta el momento, el eje central de la docencia sigue siendo la clase presencial tradicional, a pesar de que los estudiantes ya parecen técnicamente preparados para dar el salto hacia el autoaprendizaje y la generación de conocimientos. El uso de los recursos electrónicos universitarios y de las TIC en la enseñanza de la Geografía en la Universidad de Valencia está reproduciendo y consolidando la clase tradicional, pero "a distancia". Los apuntes que antes se copiaban, ahora se descargan del Aula Virtual, como se deduce de los resultados de la encuesta.

Se están reproduciendo en el nuevo contexto tecnológico los esquemas tradicionales de enseñanza-aprendizaje en los que el papel del alumno es básicamente pasivo. La inercia de la situación previa es muy poderosa, probablemente como consecuencia de las resistencias al cambio y actitudes conservadoras tanto de alumnos como de profesores, y tanto en la Universidad como en la Educación Secundaria (Solbes, Souto y Traver, 2004). En este contexto podría estar produciéndose incluso una merma en la calidad del aprendizaje: la sustitución de clases presenciales por una docencia on-line claramente pasiva no supone una mejora efectiva en el proceso de aprendizaje al tiempo que pueden perderse los aspectos positivos más interesantes de la clase presencial derivados de las dinámicas positivas de interacción personal cara a cara. De ahí la urgencia y la importancia de integrar adecuadamente el acceso a los recursos electrónicos universitarios en un nuevo esquema de aprendizaje más autónomo y creativo que aproveche plenamente sus potencialidades. Como indica Sánchez (2008), es necesaria una gestión estratégica para definir espacios en la red y conseguir que los individuos se integren e interactúen.

El estudio aquí presentado nos da una primera visión del uso de los recursos electrónicos de acceso a la información en el ámbito universitario por parte de los estudiantes, y nos revela algunas deficiencias respecto a las potencialidades didácticas que éstas presentan. Es necesario, pues, trabajar para mejorar la relación del estudiante con este tipo de recursos y para ello, resulta básico que los profesores faciliten materiales digitales adaptados a las posibilidades del multimedia interactivo.

La cultura audiovisual, hoy dominante, es especialmente interesante para la Geografía, y poco o nada utilizada en la titulación de la Universidad de Valencia. Es evidente que las redes telemáticas en la actualidad, y aún más en el futuro, ofrecen una gran cantidad de información, en distintos formatos, que precisa de un proceso permanente de análisis y actualización. En este proceso la guía del profesor es fundamental, sin ella, los estudiantes no pueden convertir dicha información en conocimiento. Esto supone la modificación del 
concepto de enseñanza y aprendizaje, que ya no es entendido como un proceso de transmisión de información, sino de construcción del conocimiento geográfico.

La formación tanto de profesores como de estudiantes en nuevas estrategias docentes usando TIC es importante para dar el paso a la cultura tecnológica que ya impera en las sociedades más avanzadas. Los estudiantes también deben aprender a usar las TIC en docencia. La mayoría tanto de profesores como de estudiantes son o han sido autodidactas en este tema y sus carencias pueden ser destacables. Por ellos, se puede afirmar, siguiendo a Del Moral y Villalustre (2009) que es importante incidir en tres temas básicos para el profesorado: el diseño técnico de los materiales didácticos, el diseño instructivo y la acción tutorial.

En cuanto a los estudiantes, objeto de este análisis, es prioritario apoyarles en el manejo del Aula Virtual y de otros recursos informáticos con el fin de desarrollar competencias relacionadas con la búsqueda, gestión, análisis, integración y evaluación de la información que encuentran en internet. Además, la red, en particular la web 2.0, favorece la comunicación y colaboración entre los estudiantes, siendo aspectos que los alumnos de Geografía, a pesar de conocer la tecnología, no han desarrollado ni se les ha ofrecido como herramienta docente. A través del Aula Virtual pueden participar en comunidades de trabajo y aprovechar de manera compartida prácticas desarrolladas en este entorno virtual, sin embargo, este tipo de participación y colaboración está muy poco desarrollado en la oferta docente de Geografía.

El estudiante presenta carencias todavía en cuanto al uso de las TIC en el aprendizaje universitario, no aprovechando al máximo las herramientas virtuales, pues en muchas ocasiones sólo ven en ellas determinadas ventajas prácticas para aprobar la asignatura. El esfuerzo pedagógico parece venir únicamente por parte del profesor, quien, por otra parte, aunque recibe cada vez más presión para incorporar las TIC en la docencia presencial, aún están infrautilizadas.

En definitiva, el desarrollo de las TIC en la docencia universitaria es básico para ofrecer un entorno de aprendizaje adecuado, autónomo y de calidad para el estudiante, quien podrá mejorar su capacidad para desarrollar su nivel de formación a lo largo de su vida, convirtiéndose en demanda potencial de estudios de nivel universitario.

El proceso de Bolonia (EEES) ha permitido reflexionar sobre el tipo de competencias que se han de valorar en los estudios de Geografía, en sus distintas materias, y sobre las herramientas pedagógicas que se pueden utilizar para conseguir la renovación de estos estudios y adaptarlos a las demandas reales de la sociedad. Es decir, como indican en su estudio Celestino, Echegaray y Guenaga (2003), las TIC por sí mismas no suponen una mejora en la calidad de la educación, a no ser que contribuyan efectivamente a la innovación pedagógica. Las TIC se configuran como una de las herramientas más potentes en este sentido, en particular por el conocimiento, potencialidad y 
buena aceptación que de ellas tienen nuestros estudiantes.

\section{Bibliografía}

ÁLVAREZ, S. et al. (2011), Actitudes de los profesores ante la integración de las TIC en la práctica docente. Estudio de un grupo de la universidad de Valladolid, Edutec-e. Revista electrónica de Tecnología Educativa, 35, http:// edutec.rediris.es/revelec2/revelec35/ (Consulta: 10 de mayo de 2011).

ANECA (2008), Informe Estudiantes. Titulados universitarios y mercado laboral. Proyecto Reflex. Agencia Nacional de Evaluación de la Calidad y Acreditación. http://www. aneca.es/var/media/151851/informes_reflex_estudiantes.pdf (Consulta: 15 de junio de 2011).

BADIA, A., PALLARES, M. y LLURDES, J.C. (2006), L'ús de les noves tecnologies en l'ensenyament de la geografía dins l'EEES. Les assignatures de Geografia Econòmica iSocial,Cartografiai fotointerpretació i SIG, en Digitum, n. 8, UOC http:// www.uoc.edu/digitum/8/cat/badia pallares_llurdes.pdf (Consulta: 1 de mayo de 2011).

BALLESTEROS, C. y LÓPEZ, E. (2003), ¿Cómo mejorar la práctica profesional de los docentes universitarios? Algunos recursos y utilidades telemáticas. Píxel-Bit, Revista de Medios y Educación, 22, 25-39.

BOSCO, A. (2005), Las TIC en los procesos de convergencia europea y la innovación en la Universidad: oportunidades y limitaciones, Aula Abier$t a, 86$, p. 3-18.

CELESTINO GUTIÉRREZ, A., ECHEGARAY LEGARRETA, O. y GUENAGA GARAI, G. (2003), Integración de las TIC en la educación superior. Píxel-Bit, Revista de Medios y Educación, 21, 21-28.

COS GUERRA, O. de; MEER LECHAMARZO, Á. de; REQUES VELASCO, P. (2011), Formación de geógrafos y aprendizaje basado en competencias: balance de un plan de innovación docente. Biblio $3 W$. Revista Bibliográfica de Geografía y Ciencias Sociales, Universidad de Barcelona, Vol. XVI, $n^{\circ} 920,25$ de abril de 2011. http://www.ub.es/geocrit/b3w920.htm. [ISSN 1138-9796] (Consulta: 5/07/2012).

DE RIBOT, M. D., LLACH, S. y CICRES, J. (2010), La incidencia de las nuevas tecnologías en los hábitos formativos de los estudiantes de Magisterio, Píxel-Bit, Revista de Medios y Educación, 37, 107-120.

DEL MORAL PÉREZ, M. E. y VILLALUSTRE MARTÍNEZ, L. (2009), Evaluación de prácticas docentes universitarias desarrolladas en entornos virtuales. Píxel-Bit, Revista de Medios y Educación, 34, 151-163.

ESTEVE, F. (2009), Bolonia y las TIC: de la docencia 1.0 al aprendizaje 2.0, $L a$ Cuestión Universitaria, 5, p. 59-68.

FREIRE, J.(2007), Los retos y oportunidades de la web 2.0 para las univer- 
sidades, en JIMENEZ, R. y POLO, F. La gran guía de los blogs, Barcelona, el Cobre, p. 82-90.

GARCÍA DE LA VEGA, A. (2010), Aplicación didáctica del aprendizaje basado en problemas al análisis geográfico, Revista de Didácticas Específicas, n. 2, p. 43-60.

GRANADOS, J. (2010), La recerca en l'ensenyament de la Geografia per al desenvolupament sostenible, Documents d'Anàlisi Geogràfica, vol, 56/2, p. 339-356.

IMBERNÓN, F., SILVA, P. y GUZMÁN, C. (2011), Competencias en los procesos de enseñanza-aprendizaje virtual y semipresencial, Comunicar. Revista Científica de Educomunicación, 36, v. XVIII, p. 107-114. DOI: 10.3916/c36-2011-03-01

JOINT QUALITY INITIATIVE (2004), Shared Dublin descriptors for short Cycle, First Cycle, Second Cycle and Third Cycle Awards, http://www.unidue.de/imperia/md/content/bologna/ dublin_descriptors.pdf (Consulta: 20 de junio de 2011).

MINISTERIO DE EDUCACIÓN Y CIENCIA (2006), Propuestas para la renovación de las metodologías educativas, http//univ.micinn.fecyt. es/univ/ccuniv/html/metodologías/ docu/PROPUESTA_RENOVACION. pdf, (Consulta: 20 de mayo de 2011).

PARLAMENTO EUROPEO Y CONSEJO EUROPEO (2006), Recomendación sobre las competencias clave para el aprendizaje permanente. http://europa.eu/legislation_summaries/education_training_youth/ lifelong_learning/c11090_es.htm (Consulta: 15 de mayo de 2011).

PÉREZTORNERO, J.M.(2008), Teacher training Curricula for Media and information Literacy. International Expert Group Meeting, UNESCO Headquarters, Paris. http:// portal. unesco.org/ci/en/files/27068/12 133527103 Background_Paper. doc/Background\%2BPaper.doc (Consulta: 1 de junio de 2011).

PRENDES, M. P. (2011), Innovación con TIC en enseñanza superior: descripción y resultados de experiencias en la Universidad de Murcia, REIFOP, 14 (1), p. 267-280, http//www.aufop.com (Consulta: 1 de septiembre de 2011).

SÁNCHEZ, J. (2008) (coord.), Comunicación y construcción del conocimiento en el nuevo espacio tecnológico. RUSC. Revista de Universidad y Sociedad del Conocimiento, América del Norte, Monográfico, 522, 10.

SÁNCHEZ, P. y ZUBILLAGA, A. (2005), Las universidades españolas ante el proceso de convergencia europeo: análisis de las medidas institucionales y acciones de aplicación y coordinación, Revista de Educación, n. 337, p. 169-187.

SANTIAGO CAMPIÓN, R. y NAVARIDAS NALDA, F. (2012), La Web 2.0 en escena. Píxel-Bit, Revista de Medios y Educación. Publicación en línea. http://sav.us.es/pixelbit (Consultada en fecha: 20 de febrero de 2012). 
SOLBES, J., SOUTO, X.M., TRAVER, M.J. (2004), El impacto de las nuevas tecnologías de la información y de la comunicación en el sistema escolar. Geo Crítica / Scripta Nova. Revista electrónica de geografía y ciencias sociales. Barcelona: Universidad de Barcelona, 1 de agosto de 2004, vol. VIII, núm. 170-71. http://www.ub.es/ geocrit/sn/sn-170-71.htm [ISSN: 11389788] (Consulta: 2 de julio de 2012).

THE NEW MEDIA CONSORTIUM (2011), Horizon Report 2011. New Media Consortium y Educase Learning Initiative, http//wp.nmc.org/ horizon2011/sections/challenges/ (Consulta: 10 de junio de 2011).
TRUJILLO, J.M. y RASO, F. (2010), Formación inicial docente y competencia digital en la convergencia europea (EEES), Enseñanza \& teaching, 28, 1-2010, 49-77.

TULLA, A. (Coord.) (2004), Libro blanco para el diseño del título de grado: Geografía y Ordenación del Territorio, ANECA, Madrid.

VERA, M.I. y PÉREZ, D. (2004), El profesorado en formación de ciencias sociales y las TICs: Situación y perspectivas, en VERA, M.I. y PÉREZ, D., Formación de la ciudadanía: las TICs y los nuevos problemas, Simposio Internacional de Ciencias Sociales, Alicante, p. 1-19. 